Copyright by the American Geophysical Union. Weimer, D. R. (2013), An empirical model of ground-level geomagnetic perturbations, Space Weather, 11, 107-120, doi:10.1002/swe.20030

\title{
An empirical model of ground-level geomagnetic perturbations
}

\author{
D. R. Weimer ${ }^{1}$
}

Received 16 November 2012; revised 18 January 2013; accepted 11 February 2013; published 28 March 2013.

[1] A new empirical model for predicting ground-level geomagnetic perturbations has been developed. This model is based on global measurements of the magnetic field at multiple stations in the Northern Hemisphere collected over an 8 year period, along with the simultaneous measurements of the interplanetary magnetic field (IMF). Variations in ionospheric conductivity are implicitly contained in the measurements used in the model's construction, including the solar $\mathrm{F}_{10.7}$ index. Provided with the IMF, solar wind velocity, dipole tilt angle (for season), and $\mathrm{F}_{10.7}$ index, this model computes all three vector components of the magnetic perturbations at specified locations. The model results are consistent with the corresponding maps of the ionospheric electric potential. Interestingly, maps of the vertical component have patterns that resemble maps of the overhead, ionospheric field-aligned currents. Comparisons of model calculations with measurements at different locations show very good results, particularly at low frequencies. There are random variations at higher frequencies that are not reproduced well with the model, but they tend to occur in proportion to the predicted levels. This model could be useful for providing regional forecasts of geomagnetic activity with an approximately $1 \mathrm{~h}$ lead time.

Citation: Weimer, D. R. (2013), An empirical model of ground-level geomagnetic perturbations, Space Weather, 11, 107-120, doi:10.1002/swe.20030

\section{Introduction}

[2] It has been long established that the geomagnetic perturbations originating from magnetospheric and auroral currents may lead to undesired effects on the ground. As outlined by Lanzerotti [2001], Boteler [2001], and Gummow [2002], geomagnetic induced currents (GIC) cause problems in man-made structures such as power transmission lines, cables, and pipelines. The variable magnetic fields can directly hinder compass navigation and surveys. Lately, these hazards have been increasingly the topic of workshops, conferences, and congressional hearings [Lanzerotti, 2012a; Turner, 2012; Langhoff and Straume, 2012].

[3] Obviously, it would be desirable to have a capability for global and localized predictions of magnetic field changes caused by space and ionospheric currents. Reliable prediction would provide time to mitigate some of the most damaging effects.

\footnotetext{
${ }^{1}$ Center for Space Science and Engineering Research, Bradley Department of Electrical and Computer Engineering, Virginia Tech, Blacksburg, Virginia, USA.

Corresponding author: D. R. Weimer, Virginia Tech, National Institute of Aerospace, 100 Exploration Way, Hampton, VA 23666, USA. (dweimer@vt.edu)
}

(02013. American Geophysical Union. All Rights Reserved. 1542-7390/13/10.1002/swe.20030
[4] There has been progress toward a predictive capability using various techniques. One method uses numerical, "first-principle," computer simulations of the coupled solar wind-magnetosphere-ionosphere system. The publications on this topic are numerous; the papers by De Zeeuw et al. [2004], Raeder et al. [2001], Wiltberger et al. [2004], and Pulkkinen et al. [2007] are a few examples. Other methods for the forecast of geomagnetic perturbations use nonlinear filters [Gleisner and Lundstedt, 2001; Weigel et al., 2002] or empirical, ionosphere models [Weimer, 2005].

[5] Hall currents in the ionosphere are the primary source of the geomagnetic disturbances that are seen at the surface of the Earth, particularly at high latitudes (L. Rastätter et al., Community-wide Geospace Model validation: Computation of $\Delta B$ at the CCMC, manuscript in preparation, 2012). The physics-based models require values of the Hall conductivity in order to obtain the Hall currents from the electric potentials and fields that they calculate; they also require values of the Pedersen conductivity in order to obtain the relationships between the electric fields and the field-aligned currents going to and from the magnetosphere. These conductivities are generally obtained from models or may be explicitly calculated from mathematical formulas and some knowledge of particle fluxes. From the Hall currents, the magnetic perturbations are then derived from application of the Biot-Savart law. Large uncertainties in the conductivity distribution are presently the weakest component in such methods. 
[6] In the case of the empirical method described by Weimer [2005], ionospheric models of electric potentials and field-aligned currents are used together in order to obtain the Hall currents. With this method, it is necessary to assume a fixed ratio between the Hall and Pedersen conductivity, which is the primary disadvantage, but it still works fairly well for the large-scale variations.

[7] One difficult aspect of the geomagnetic calculations that are rooted in the ionosphere is the effect of currents that are induced underground [Pirjola and Viljanen, 1998; Tanskanen et al., 2001; Pulkkinen et al., 2007]. The calculation of the induced currents is further complicated by "Earth conductivity structures that are not well mapped" [Lanzerotti, 2012b]. The simplest solution is to assume that the image currents are at a fixed depth below the surface.

[8] A different method for calculating global magnetic perturbations is to use an empirical model that is constructed from global, ground-based magnetometer measurements, along with the simultaneous measurements of the solar wind and interplanetary magnetic field (IMF). A model derived in this manner would implicitly combine the effects of the electric fields, seasons, and variable conductivity in the ionosphere, as well as the effects of the underground image currents. Therefore, this method should be more accurate than any predictions that are based on ionospheric currents, as it bypasses assumptions and estimations that are required.

[9] The purpose of this paper is to describe one such model. A prior publication by Weimer et al. [2010] (hereafter referred to as W10) outlined the preliminary steps toward this model's development. The IMF and magnetic field data sources and their processing were described in detail, as well as tests to determine the delay between the time of arrival of the solar wind at the bow shock and the time that effects are seen on the ground. In the W10 paper, it was demonstrated that, by selecting a subset of global magnetic field measurements according to the solar wind velocity, IMF, and tilt angle (within a range of values), there resulted coherent patterns in the magnetic perturbations when plotted in geomagnetic coordinates. Least squares fits of spherical cap harmonic analysis (SCHA) [Haines, 1985] coefficients were used to generate maps of these magnetic perturbations for the selected conditions, and these maps were shown side by side with scatter plots of the original data points. The development of the actual empirical model followed later. This model has the capability to produce maps of each of the three vector components of the geomagnetic perturbations, given a specification of solar wind velocity, IMF values, dipole tilt angle, and the solar $\mathrm{F}_{10.7}$ index.

[10] Performance tests are beyond the scope of this paper and will not be discussed in great detail. To respond to the need for a quantitative and systematic evaluation of different predictive techniques, the Community Coordinated Modeling Center (CCMC) has taken on the role of evaluating model submissions. Pulkkinen et al. [2011] and A. Pulkkinen et al. (Community-wide geospace model validation to support model transition to operations, submitted to Space Weather, 2012) describe the initial results of evaluating the prediction performance of several different methods during selected time intervals, including earlier versions of this empirical model.

\section{Data Sources}

[11] This project uses ground-level vector magnetic field measurements from stations in the Northern Hemisphere. The majority of data were obtained from the International Real-time Magnetic Observatory Network (INTERMAGNET) [St-Louis, 2008; Rasson, 2007]. Additional sources of data are the Danish Greenland chain, the Canadian Array for Realtime Investigations of Magnetic Activity (CARISMA, previously known as CANOPUS), the Augsburg College Magnetometer Array for Cusp and Cleft Studies (MACCS) [Engebretson et al., 1995; Hughes et al., 1995], Japan's “210 MM Magnetometer Network" [Yumoto and CPMN Group, 2001], the European International Monitor for Auroral Geomagnetic Effects (IMAGE), and the Geophysical Institute Magnetometer Array (GIMA).

[12] The W10 publication describes in detail the magnetometer data acquisition and the preprocessing steps that were required prior to use for this project. These processing steps included the subtraction of baseline values that varied over time. Different types of ground stations used different coordinate systems, either local magnetic or geographic, and they also required different methods for handling the offsets. After offset subtraction, all magnetic perturbation vectors were then rotated into a corrected geomagnetic (CGM) coordinate system. Magnetic apex coordinates [VanZandt et al., 1972] are used, or more specifically, the "Modified Apex" coordinates described by Richmond [1995] and Emmert et al. [2010]. At high, auroral latitudes, these apex coordinates are nearly identical to the Altitude Adjusted Corrected Geomagnetic system described by Baker and Wing [1989]. At lower latitudes, the modified apex coordinates are always valid, whereas corrected geomagnetic coordinates have locations with undefined mappings [Gustafsson et al., 1992]. Magnetic local time (MLT) is calculated from the hour angle from the subsolar position in modified apex coordinates. For convenience, the CGM acronym is used here to refer to Modified Apex coordinates.

[13] The database discussed in W10 had a duration of almost 4 years, up through 2001. Afterward, the duration of the processed data had been extended by another 4 years, so that the total duration of the database now spans a nearly 8 year time period, from February 1998 thru December 2005. The processing procedures for the second 4 year period was the same as before. A small number of magnetometer stations having some data prior to 2002 had ceased operation, while several new stations were added. In all, data had been processed from 149 different locations at modified apex latitudes greater than zero. The majority of sites, at apex latitudes over $40^{\circ}$, are tabulated in the W10 publication.

[14] It is well known that the distribution of magnetic observatories has gaps in geographic coverage, particularly 
in the Alaska-Russian sector near the pole. But this model only uses the MLT of each measurement, not caring about the geographic longitude. As demonstrated in W10, due to the rotation of the Earth, each station samples all local times, effectively filling in gaps in both longitude (MLT) and latitude during the 8 year time period.

[15] The interplanetary space environment measurements required for the model development are from NASA's Advanced Composition Explorer (ACE). "Level 2" data were obtained from the Magnetic Field Instrument [Smith et al., 1998] and the Solar Wind Electron, Proton, and Alpha Monitor (SWEPAM) [McComas et al., 1998] instruments. These "upstream" solar wind measurements, taken at approximately $240 R_{E}$ ahead of the Earth, were propagated to the nose of the bow shock, at $X=14.6 R_{E}$ GSE [Fairfield, 1971]. The technique described by Weimer and King [2008] was used to calculate the time delays, accounting for variability in the orientation of the IMF phase fronts. The beginning of the processed ACE data in early February 1998 determined the starting date of the combined database.

[16] The interplanetary measurements and the groundbased magnetic perturbations from every site were all reduced to $5 \mathrm{~min}$ averages at $5 \mathrm{~min}$ intervals. In W10, it had been found that the best correlation (0.82) between the IMF and $5 \mathrm{~min}$ ground data is obtained if $25 \mathrm{~min}$ averages are used for the IMF. The same timings are used in this final model development, with IMF averaging windows, as timed at the bow shock, starting and ending at 45 and $20 \mathrm{~min}$ before the start of the corresponding delta-B samples on the ground. The time delay between the IMF and ground response is assumed to account for propagation of the IMF from the bow shock to magnetosphere, followed by propagation of signals to ionosphere, as well as the nonzero reconfiguration time of the global ionosphere.

[17] The geomagnetic disturbances on the ground are influenced by ionospheric conductivity, which is controlled in part by solar ultraviolet radiation levels. Therefore, this model includes the $\mathrm{F}_{10.7}$ index as a measure of solar radiation. The $\mathrm{F}_{10.7}$ values used in this project were obtained from tables maintained by K. Tobiska at www.spacewx.com [Tobiska et al., 2008]. The decision to use a time period of 8 years for the database was motivated by a desire to sample a range of solar variability over the major part of a solar cycle, as well as in obtaining more samples during times with more extreme IMF magnitudes.

\section{Empirical Model Construction}

[18] The model is designed to calculate a value for each of the three magnetic vector components as a function of CGM latitude and MLT, given a specification of IMF conditions. SCHA, described by Haines [1985], is used to generate a mapping function for each component. Earlier, development versions of this model had used spherical cap "half angles" of less than $90^{\circ}$, where the Associated Legendre polynomials have degrees that are real (fractional) numbers that depend on an integer index as well as both the integer order and polar cap half angle [Haines, 1985]. In this latest model version, the size of the spherical cap is extended to $90^{\circ}$ so that the low-latitude boundary is at the geomagnetic apex equator and the Legendre polynomials have their standard integer degrees. Only even pairings of degree, $l$, and order $m$ are used, forcing a zero slope at the low-latitude boundary [Haines, 1985]. This reduces unnatural oscillations at the lower latitudes, where the data are more sparse.

[19] In equation form, each vector component in the model is represented with

$$
\Delta B_{X}(\Lambda, \phi)=\sum_{l=0}^{31} \sum_{m=0}^{3<l} P_{l}^{m}(\cos \Lambda)\left(g_{l}^{m} \cos m \phi+h_{l}^{m} \sin m \phi\right)
$$

where $\Delta B_{X}$ represents either the north-south, east-west, or vertical geomagnetic perturbation. The angle $\Lambda$ represents the CGM colatitude, and $\phi$ is the MLT in radians.

[20] In the empirical model, each of the $g_{l}^{m}$ and $h_{l}^{m}$ coefficients in (1) need to smoothly vary as a function of the IMF, solar wind velocity, dipole tilt angle, and $F_{10.7}$ index. The model needs to reproduce the variations in the magnetic field that result from changes in the ionosphere that occur when the IMF varies in magnitude and orientation in the GSM $Y-Z$ plane. Rather than use linear functions of the IMF $B_{Y}$ and $B_{Z}$ directly, prior experience with electric potential models has indicated that the nonlinear response of the ionosphere is reproduced well with a Fourier series that uses the IMF clock angle, $\theta_{c}$. This Fourier series needs a maximum order of only two to reproduce the nuances in the clock angle variations.

[21] Following trials with numerous permutations of the model inputs variables and calculation of coefficients, the best model performance up to now is obtained when the $g_{l}^{m}$ and $h_{l}^{m}$ coefficients are calculated using the following:

$$
g_{l}^{m}=c_{0}+c_{1} B_{T}+c_{2} V_{S W}+c_{3} t+c_{4} \sqrt{F_{10.7}}+
$$

$c_{5} B_{T} \cos \left(\theta_{c}\right)+c_{6} V_{S W} \cos \left(\theta_{c}\right)+c_{7} t \cos \left(\theta_{c}\right)+c_{8} \sqrt{F_{10.7}} \cos \left(\theta_{c}\right)+$ $c_{9} B_{T} \sin \left(\theta_{c}\right)+c_{10} V_{S W} \sin \left(\theta_{c}\right)+c_{11} t \sin \left(\theta_{c}\right)+c_{12} \sqrt{F_{10.7}} \sin \left(\theta_{c}\right)+$ $c_{13} B_{T} \cos \left(2 \theta_{c}\right)+c_{14} V_{S W} \cos \left(2 \theta_{c}\right)+c_{15} B_{T} \sin \left(2 \theta_{c}\right)+c_{16} V_{S W} \sin \left(2 \theta_{c}\right)$

$B_{T}$ is the magnitude of the tangential IMF in the GSM $Y-Z$ plane, having a "clock angle" of $\theta_{c}$. $V_{S W}$ is the solar wind velocity, and $t$ is the dipole tilt angle expressed in radians, which at small values are proportional to $\sin (t)$. This tilt is the angle that defines the transformation between the GSM and SM coordinate systems, through a rotation around their common $Y$ axis [Hapgood, 1992, 1997]. The tilt angle is zero at the equinoxes and $\pm 23.3^{\circ}$ at solstices, with a daily variation or "wobble" of approximately $\pm 10^{\circ}$ superimposed, due to the offset between the geographic and geomagnetic poles. The $\mathrm{F}_{10.7}$ index represents ionosphere conductivity variations due to solar ultraviolet radiation and is expressed as solar flux units (sfu). In (2), the square root of $\mathrm{F}_{10.7}$ is used, following a formula for ionospheric conductivity by Robinson and Vondrak [1984].

[22] The formulas used for all $h_{l}^{m}$ are the same as in (2), and for every $l-m$ pair, each $g_{l}^{m}$ and $h_{l}^{m}$ have their own set 
of $17 c_{n}$ coefficients. Least squares fits were used to derive the values of all $c_{n}$ coefficients. The fits were accomplished by expressing the solution in a linear matrix equation of the form $\mathbf{A} \cdot \mathbf{x}=\mathbf{b}$ that is solved by the method of "LU decomposition" [Press et al., 1992]. The three vector components of the magnetic field have separate solutions, but they share common matrices that are reused for greater efficiency. The programming for these computations used the Interactive Data Language and one subroutine in FORTRAN. From prior experience, the author had found that numerical overflow or underflow can occur in the LU decomposition if some matrix elements are 2 orders of magnitude larger than the others. In order to avoid this problem, the terms containing the solar wind velocity, in units of kilometers per second, were divided by 100 .

[23] It is known that ionospheric electrodynamics responds to increasing levels of driving by the solar wind/IMF in a nonlinear manner [Siscoe et al., 2002; Kivelson and Ridley, 2008]. In order to model the expected, nonlinear response with linear fits, the data are subdivided into 29 bins, sorted according to the magnitude of the IMF $B_{T}$. Separate, linear solutions, as in (2), are obtained using the data within each IMF bin in the datafitting process. A smoother response curve (as shown in the next section) was obtained by overlapping the data in adjacent bins when doing the fits. For the final model calculation at a specified IMF $B_{T}$ magnitude, the $c_{n}$ coefficients are interpolated between bins, followed by the application of (2) and then (1).

[24] A graph that shows the number of data samples ( $25 \mathrm{~min}$ averages at $5 \mathrm{~min}$ increments over 8 years) in each bin, arranged by IMF magnitude in the GSM $Y-Z$ plane, is shown in Figure 1. The IMF magnitudes have an uneven distribution, so that the majority of time samples are

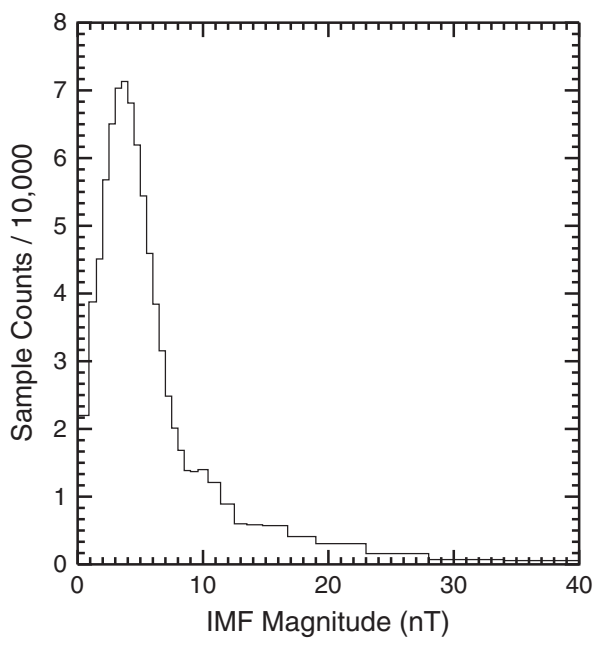

Figure 1. Distribution of IMF samples by magnitude. The number of sample counts (divided by 10,000) are shown for each bin. The width of each bin increased above $9 \mathrm{nT}$, as shown on the plot. These are $25 \mathrm{~min}$ averages at $5 \mathrm{~min}$ increments, over an 8 year period. clustered under $5 \mathrm{nT}$. The number of samples gets progressively smaller as the IMF magnitude increases. In the range of IMF magnitudes between 1.5 and $9.0 \mathrm{nT}$, the bins are $0.5 \mathrm{nT}$ wide; above $9 \mathrm{nT}$, the bins sizes were adjusted so that they steadily increased in width in order to produce more reasonable data counts in each bin, although the counts are still decreasing as the IMF magnitude increases. There are relatively few times having IMF $B_{T}$ magnitudes greater than $20 \mathrm{nT}$, even though the most critical space weather events are in this range. An earlier version of the model had used bins having equal data counts, but the uppermost bin contained everything above approximately $14 \mathrm{nT}$ and could not adequately resolve the saturation response curve.

[25] Solar wind number density and dynamic pressure are not included in (2). These variables had been tried during the early stages of the model development, and it had been found that their inclusion did not have much of any effect on the model accuracy. The reason is the magnetosphere seems to respond only to sudden changes in the density and pressure. For example, Ober et al. [2007] found that the transpolar, ionospheric electric potential has a transient response to sudden changes in density, "decaying to near initial steady state values even when the density change persists." Other examples of temporal changes in the potentials, derived from magnetic recordings and lasting only $10-30 \mathrm{~min}$, are shown by Stauning and Troshichev [2008]. As this empirical model shows the mean or steady state response to specified IMF conditions, it cannot resolve such nonpersistent, temporal changes.

[26] Other trials early in the model's development had used terms with the product $B_{T} V_{S W}$ in an alternative version of (2), rather than using the separate terms shown in (2). Although the former combination, which represents the solar wind electric field, was expected to work the best, the test results favored the version shown in (2).

[27] It would seem that, since the polar cap potential drop varies approximately as $\sin ^{2}\left(\theta_{c} / 2\right)$ [Sonnerup, 1974], it would be possible to use this function rather than a Fourier series in (2). But $\sin ^{2}\left(\theta_{c} / 2\right)$ is symmetric and would not reproduce the subtle shifts in the large-scale mappings of the electric potentials and currents that are observed when IMF $B_{Y}$ changes sign. More problematic is the reproduction of the patterns for a Northward IMF, as the clock angle and this function are zero.

[28] In (1) the maximum degree $l$ is as high as 31 in order to fully resolve the peak values of the magnetic perturbations, particularly for the small but intense spatial features found at high latitudes with a northward $(+Z)$ IMF. No further improvement in model accuracy is obtained with $l$ values over 31 or $m$ greater than 3 .

\section{Example Model Results}

[29] To show examples of the new model's output, Figure 2 shows contour maps of the north-south magnetic perturbations that result when the IMF is purely southward. The values of IMF $B_{Z}$ are $-2 \mathrm{nT},-6 \mathrm{nT}$, and $-18 \mathrm{nT}$, with the magnitude increasing by a factor of three in each step. 

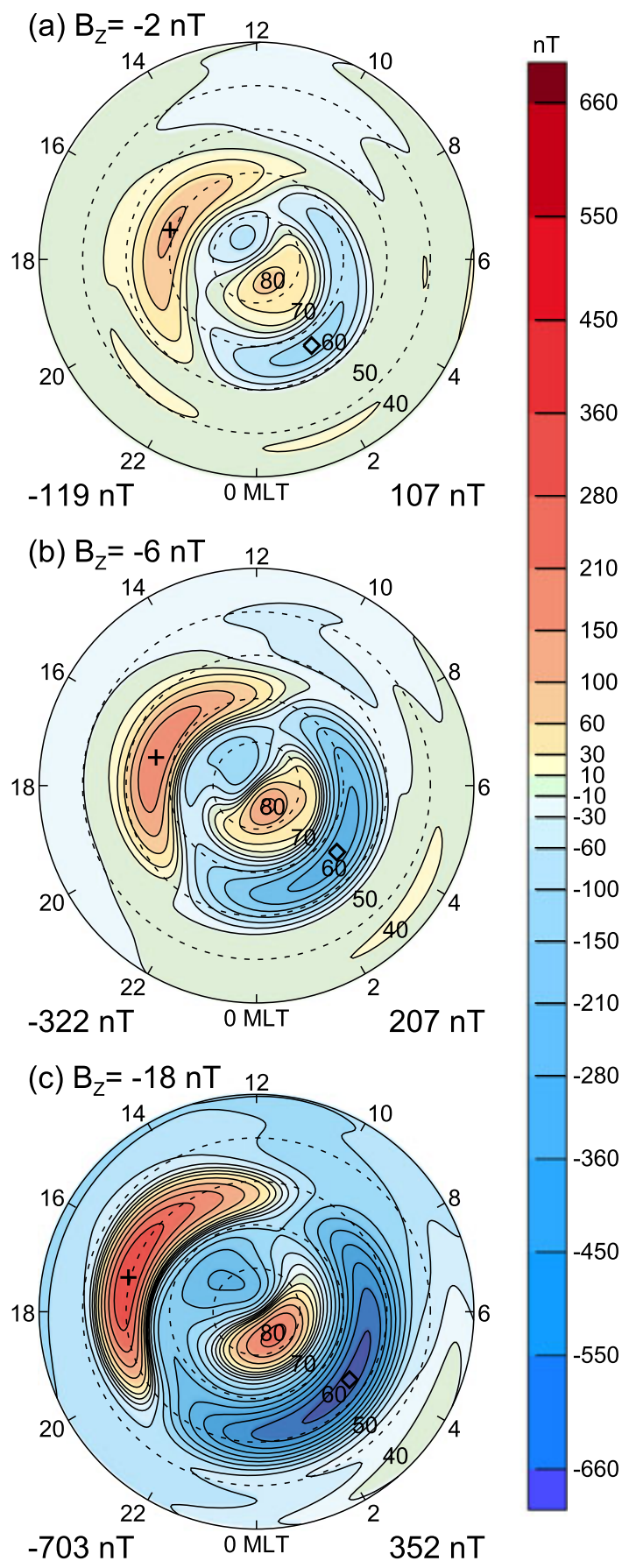

Figure 2. Maps of $\Delta B_{\text {North }}$ for southward IMF at three magnitudes. (a) IMF $B_{Z}=-2 \mathrm{nT}$, (b) IMF $B_{Z}=-6 \mathrm{nT}$, and (c) IMF $B_{Z}=-18 \mathrm{nT}$.

The other input parameters are $450 \mathrm{~km} \mathrm{sec}^{-1}$ for solar wind velocity, a dipole tilt angle of $0^{\circ}$, corresponding to equinox, and the $\mathrm{F}_{10.7}$ index is $120 \mathrm{sfu}$. These maps are in geomagnetic apex coordinates and MLT. In each map, the locations of the minimum and maximum values are marked with the "diamond" and "plus" symbols, and the values of these peaks are indicated in the lower left and right corners of each map. The contour levels have larger separations as the magnitudes increase in order to better show the variations over the entire range of magnitudes. Although the model goes down to the geomagnetic equator, these maps have a lower latitude limit of $40^{\circ}$ in order to show the higher latitudes with a better resolution.

[30] As is expected, the latitude range of the geomagnetic variations move toward the equator as the magnitude of the southward IMF increases. For example, the peak northward perturbation located between 16 and 17 MLT is positioned near $60^{\circ}$ latitude in Figure $2 \mathrm{a}$, and it is at $50^{\circ}$ latitude in Figure 2c. An interesting feature shown in Figure 2 is that there is a southward (negative) perturbation at subauroral latitudes that becomes more prominent as the IMF grows more southward. It appears that the model is reproducing the perturbations from the ring current that are monitored with the Dst index.

[31] Figure 3 shows how the northward geomagnetic field responds as the IMF increases in magnitude. This response is shown for IMF clock angles of $0^{\circ}, 9^{\circ}, 180^{\circ}$, and $270^{\circ}$, arranged in this order from top to bottom. This graph illustrates how the division of the data into bins provides for a replication of the nonlinear saturation effects, while using linear fits within small sections of the curve. The solid blue line indicates the absolute values of the most negative perturbation, and the solid red line shows the peak positive values for a solar wind velocity of $450 \mathrm{~km} \mathrm{sec}^{-1}$. The dipole tilt angle and $\mathrm{F}_{10.7}$ index are again at $0^{\circ}$ and $120 \mathrm{sfu}$, respectively. The dotted lines show the same variations when the solar wind velocity is $300 \mathrm{~km}$ $\sec ^{-1}$, and the longer, dashed lines show the results for $600 \mathrm{~km} \mathrm{sec}^{-1}$.

[32] The purpose of these graphs is to show the effects of the nonlinear saturation of the polar cap potentials as the solar wind/IMF driving increases [Siscoe et al., 2004], particularly for the southward orientation $\left(180^{\circ}\right)$. In Figure $3 c$, it can be seen that the positive, northward perturbations saturate sooner, and at a much lower magnitude, than the negative perturbations. It is also evident that, at the southward orientation, changes in the solar wind velocity also affect the negative perturbations (blue lines) more than the positive values (red lines). As shown in Figure 2, with a southward IMF the peaks in the positive perturbations are generally located post-noon at about 17 MLT, and the most negative values are found near 4 MLT.

[33] In Figure 3, the magnitude of the perturbations at some clock angles have sharp, upward turns where the magnitude of the IMF is above the $30 \mathrm{nT}$ level. These discontinuities in the slope are typically more pronounced for the negative values (blue lines) than the positive values (red lines). It is suspected that these features are artifacts that result from the very small number of samples obtained at these large IMF values, as shown in Figure 1. The majority of measurements above $40 \mathrm{nT}$ are from the well-known geomagnetic storm periods in OctoberNovember 2003. 
(a) IMF Clock Angle $=0^{\circ}$

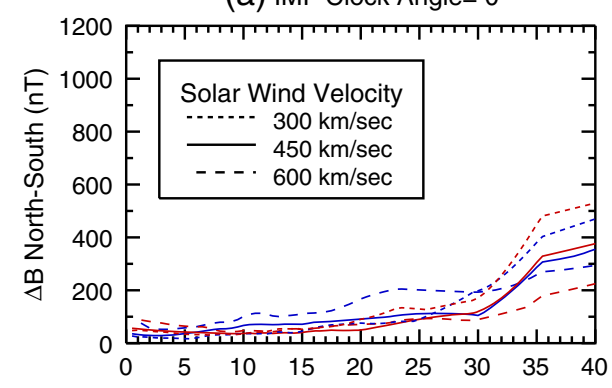

(b) IMF Clock Angle $=90^{\circ}$

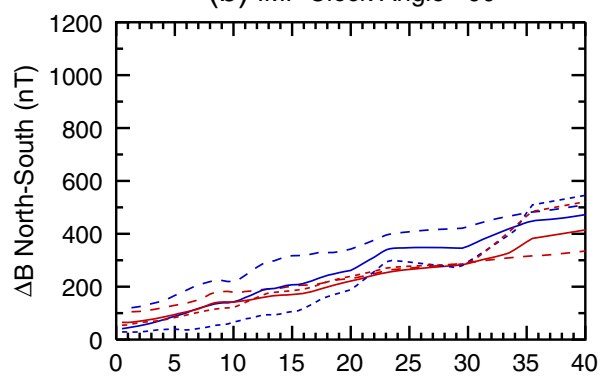

(c) IMF Clock Angle $=180^{\circ}$

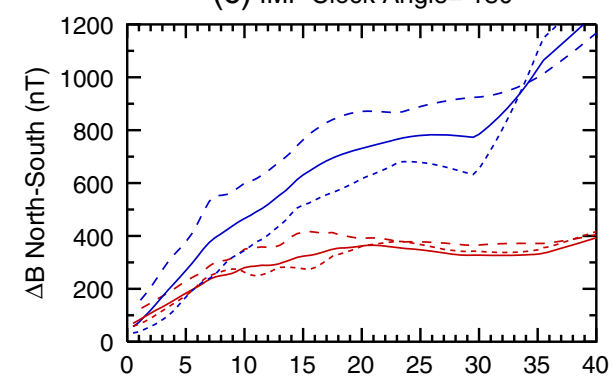

(d) IMF Clock Angle $=270^{\circ}$

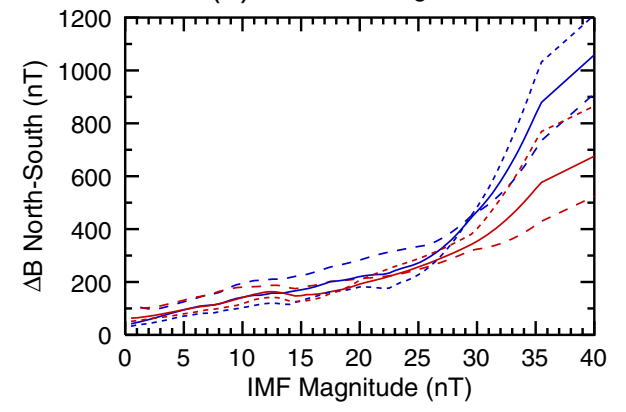

Figure 3. Graph of minimum and maximum $\Delta B_{\text {North }}$ as a function of IMF magnitude, at clock angle orientations of (a) $0^{\circ}$, (b) $90^{\circ}$, (c) $180^{\circ}$, and (d) $270^{\circ}$. The red lines in these graphs show the value of the maximum $\Delta B_{\text {North }}$ magnetic perturbations as a function of IMF magnitude, and the blue lines show the absolute value of the minimum $\Delta B_{\text {North }}$. The solid lines show the results with the solar wind velocity set at $450 \mathrm{~km} \mathrm{sec}^{-1}$, and the dotted and dashed lines show the results when the velocity is changed to 300 and $600 \mathrm{~km} \mathrm{sec}^{-1}$. The dipole tilt was set to zero, corresponding to the equinox, and $\mathrm{F}_{10.7}$ was $120 \mathrm{sfu}$.
[34] Additional examples of magnetic perturbation contour maps are shown in Figure 4. There is one column for each of the three vector components, north-south, east-west, and vertical. The four rows illustrate the results for IMF orientations at each of four basic directions in the GSM Y-Z plane. The magnitude of the IMF is $8 \mathrm{nT}$, and the solar wind velocity is again at the mean value of $450 \mathrm{~km} \mathrm{sec}^{-1}$.

[35] At low latitudes, the "solar quiet daily variation," also known as the $S_{q}$ field [Knecht and Shuman, 1985], is expected to be present. Figure 5 shows the three components of the magnetic variations, from the new empirical model, as a function of MLT at geomagnetic apex latitudes of $5^{\circ}$ and $50^{\circ}$. These are graphed for quiet conditions, with an IMF magnitude of $1 \mathrm{nT}$ in the northward direction and a $300 \mathrm{~km}$ $\mathrm{sec}^{-1}$ solar wind velocity. The red line is the result with an $\mathrm{F}_{10.7}$ index of $240 \mathrm{sfu}$, and the blue line shows results with an $\mathrm{F}_{10.7}$ index of $80 \mathrm{sfu}$ and again for zero tilt. The vertical scale for the northward component is slightly more than double the scale for the other two components. There is an excellent agreement with the $S_{q}$ values shown by Knecht and Shuman [1985] in their Figure 4-21, and Figure 5b by Matsushita and Maeda [1965]. As already mentioned, the equatorial field becomes more southward as the geomagnetic activity increases.

[36] Figures 6, 7, and 8 contain an example of a comparison between model results with actual magnetometer measurements. These graphs show the three vector components at 11 stations during a very active, 6 day interval from 12:00 UT on 22 July 2004 through 12:00 UT on 28 July 2004 . The blue lines show the magnetic perturbations calculated with the model, and the red lines show the measured magnetic fields minus the monthly mean values. The three-letter International Association of Geomagnetism and Aeronomy (IAGA) codes for the magnetometer stations are indicated by the left and right axes. Their locations, which are tabulated in the W10 publication, span a wide range in longitude. Only IMF and solar wind measurements from the ACE satellite, as well as the $F_{10.7}$ index and tilt angles, are used as input for the model. These data are plotted in geographic "XYZ" coordinates, as the majority of magnetic observatory measurements are provided in this system. The horizontal (northward and eastward) model outputs were rotated to align with the geographic axes. The angles of rotation vary with geographic location and are derived from the geographic to geomagnetic apex transformations. The vertical model results required no rotation.

[37] While the visual comparisons in these prior figures are useful, it is also informative to look at other evaluations of the model's performance. In the interest of brevity, only a subset of mean errors over the time span of the input data will be shown. To begin with, for purpose of comparison, graphs in Figure 9 show the mean absolute value of the measured magnetic perturbations as a function of corrected geomagnetic apex latitude. For this calculation, the entire 8 year database was used, at $5 \mathrm{~min}$ increments. For this graph, the times were restricted to only those where the magnitude of the measured IMF was in the range of 12 to $20 \mathrm{nT}$, with no sorting by IMF clock angle. The plots in Figures 9a-9h show results for sorting by the 


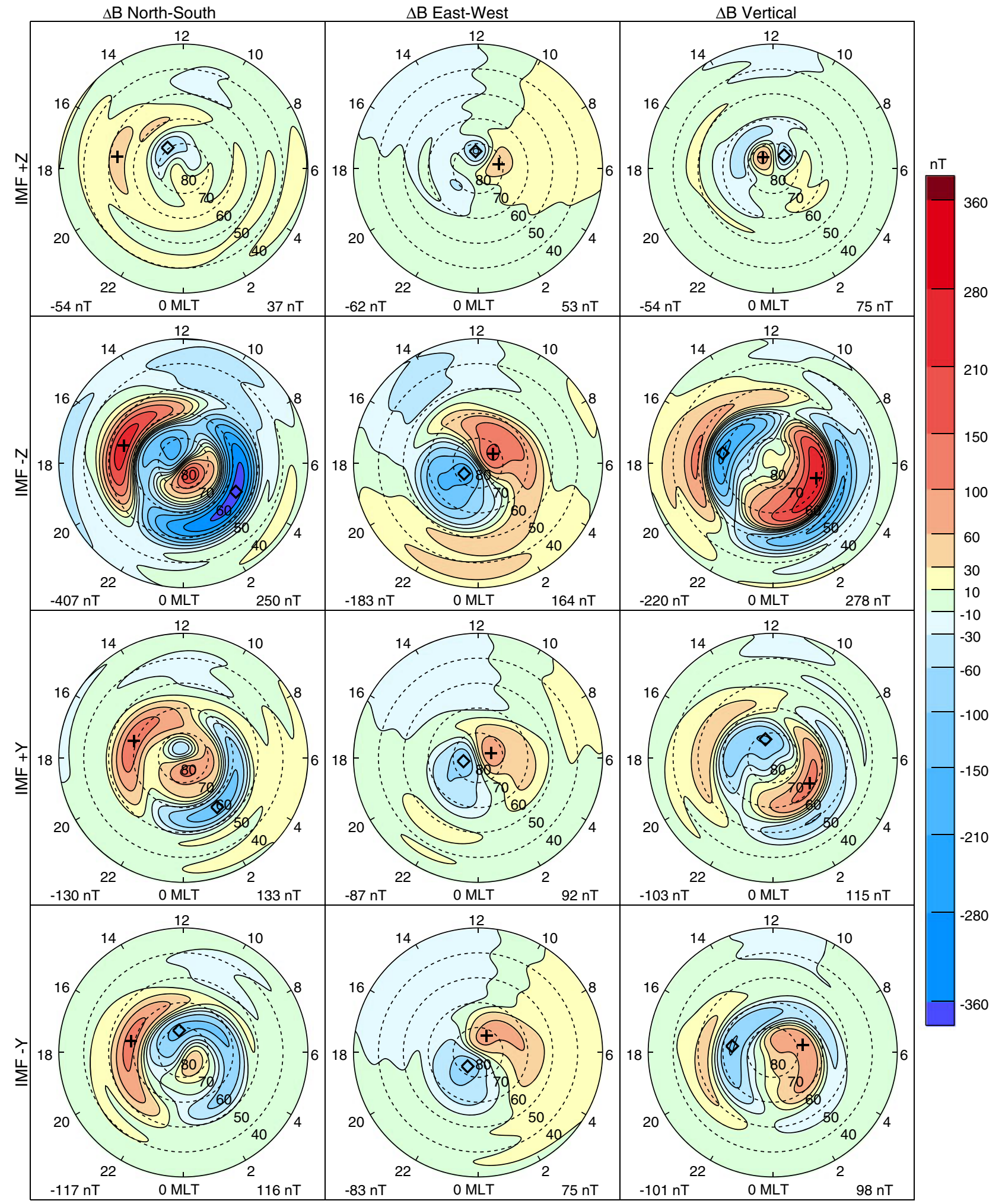

Figure 4. Maps of $\Delta B_{\text {North }}$ (left), $\Delta B_{\text {East }}$ (middle), and $\Delta B_{\text {Vertical }}$ (right) at four IMF orientations. The IMF magnitude is $8 \mathrm{nT}$, and the four rows show the results for the $+Z,-Z,+Y$, and $-Y$ GSM orientations. 



Figure 5. Graphs of $\Delta B_{\text {North }}$ (left), $\Delta B_{\text {East }}$ (middle), and $\Delta B_{\text {Vertical }}$ (right) versus MLT. These are shown for quiet conditions with a weakly northward IMF, at CGM apex latitudes of $5^{\circ}$ and $50^{\circ}$. The red lines show the results with $\mathrm{F}_{10.7}=240 \mathrm{sfu}$, and $\mathrm{F}_{10.7}=80 \mathrm{sfu}$ in the blue lines.



Figure 6. Graph of $\Delta B_{\text {North }}$ versus time. Model calculations using IMF values are shown with the blue lines, at 11 locations indicated with their three-letter IAGA codes. Measurements are shown with the superposed red lines, after baseline subtraction. The plot begins at 12:00 UT on 22 July 2004 and ends at 12:00 UT on 28 July 2004. The sites are ordered from top to bottom by corrected geomagnetic apex latitude.

measurements according to each station's MLT at every time step, using eight bins that span $3 \mathrm{~h}$ of MLT. The three lines show the results for $\Delta B_{\text {North }}$ (solid), $\Delta B_{\text {East }}$ (dotted), and $\Delta B_{\text {Vertical }}$ (dashes). Each data point in a line corresponds to one site, although not every site had collected data for the entire 8 years. The variability in the lines are due to the fact that the various sites were not in the same MLT bins during the same times, which varied by clock angle, solar wind velocity, and season. Another source of variability is due to the need to use data from magnetometers that are not true "observatories." As described in the W10 paper, some variometer instruments are not as accurate or stable over long time periods, in comparison to the observatory-class stations. Nevertheless, in Figure 9 the auroral zone is easily visible in the plots showing data taken near midnight, such as Figures 9a and 9h. Different characteristics are evident in the data obtained near local noon, such as Figures $9 \mathrm{~d}$ and $9 \mathrm{e}$. 


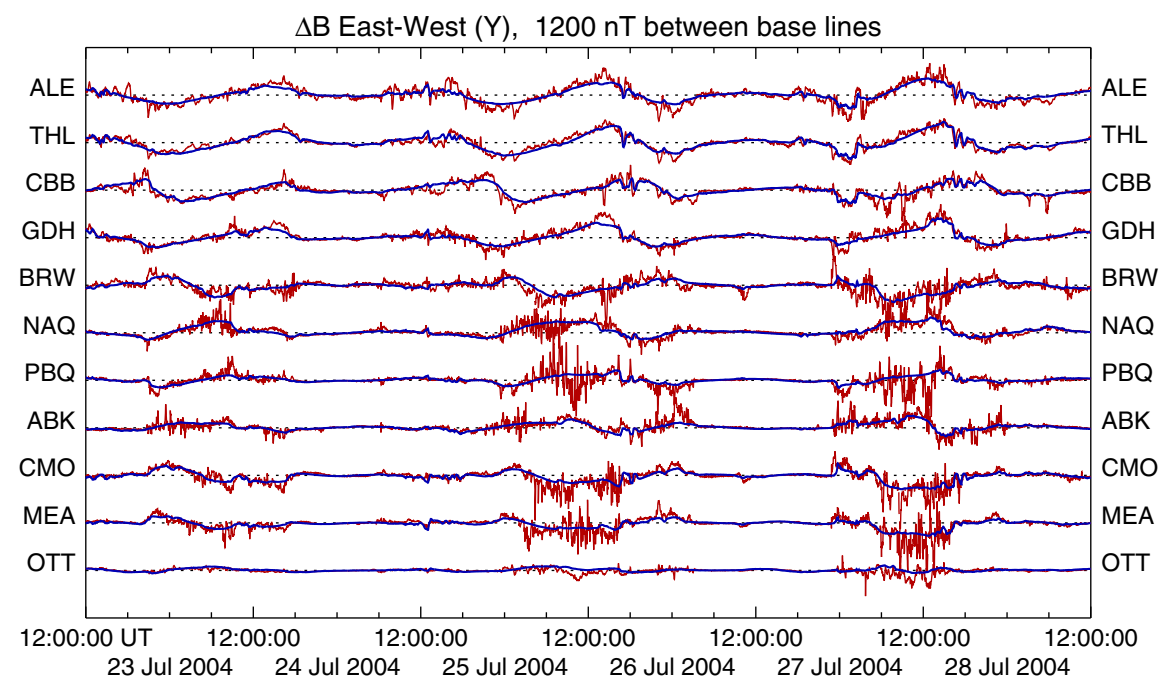

Figure 7. Graph of $\Delta B_{\text {East }}$ versus time. The format and time ranges are the same as in Figure 6.

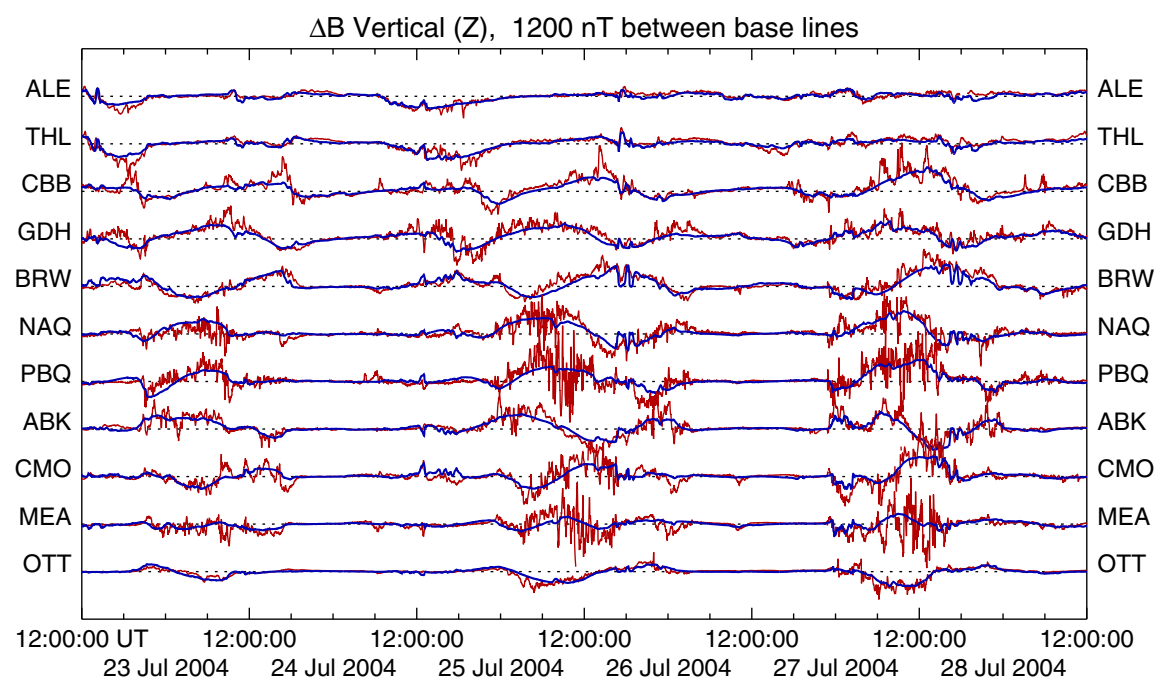

Figure 8. Graph of $\Delta B_{\text {Vertical }}$ versus time. The format and time ranges are the same as in Figure 6.

[38] Next, in Figure 10 are shown the mean of the absolute value of model errors, using the same subset of data and same plot format. At every 5 min time step, the actual IMF, solar wind velocity, $\mathrm{F}_{10.7}$ index, and dipole tilt angle were input to the model. The differences between the model output and measured magnetic perturbations were calculated at each site to obtain these means, using the absolute values. As in (9), the three lines show the results for $\Delta B_{\text {North }}$ (solid), $\Delta B_{\text {East }}$ (dotted), and $\Delta B_{\text {Vertical }}$ (dashes). It seems apparent that these errors are roughly proportional to the mean values. The northward and eastward components have the largest errors, on the order of $50 \%$ to $60 \%$ of the mean values. As seen in Figure 6, the differences between the model predictions and measurements depend largely on the temporal frequency of the variations, and these fluctuations tend to have magnitudes that are on the same order as the underlying, baseline values.

\section{Discussion}

[39] The results shown in Figures 6-8 show that this empirical model does a very good job at calculating the levels of the geomagnetic perturbations at the lower frequencies, where the perturbations are strongly influenced by the IMF. The higher-frequency fluctuations are not reproduced so well; the figures show that these fluctuations 


\section{WEIMER: A MODEL OF GEOMAGNETIC PERTURBATIONS}

(a) $\mathrm{MLT}=0-3$ hours

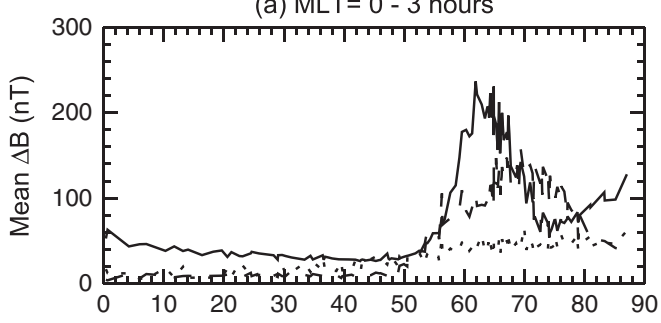

(c) $\mathrm{MLT}=6-9$ hours

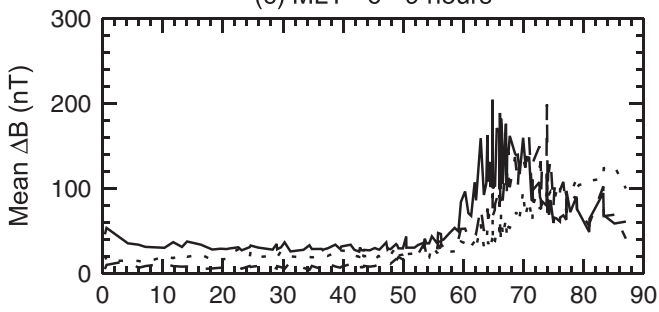

(e) $\mathrm{MLT}=12-15$ hours



(g) $\mathrm{MLT}=18-21$ hours

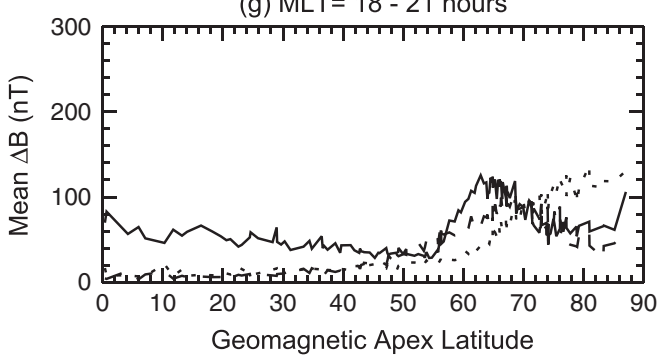

(b) $\mathrm{MLT}=3-6$ hours

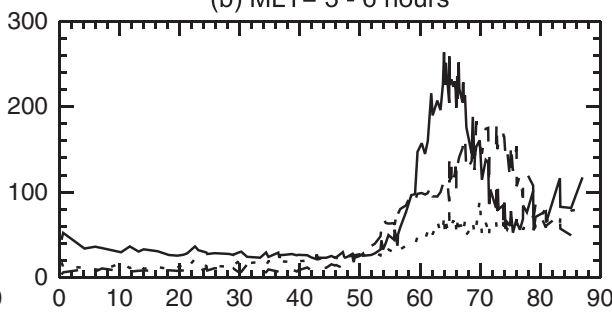

(d) $\mathrm{MLT}=9-12$ hours

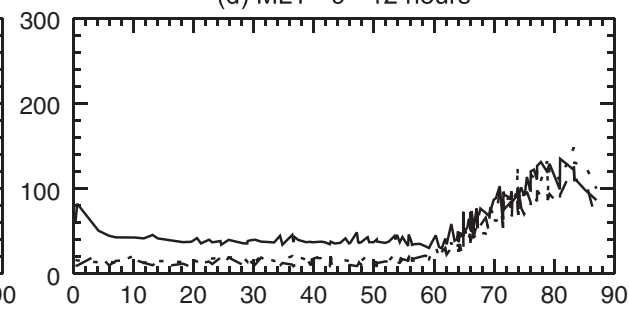

(f) $\mathrm{MLT}=15-18$ hours

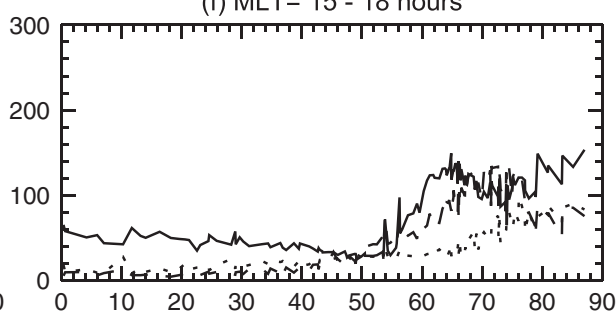

(h) $\mathrm{MLT}=21-24$ hours

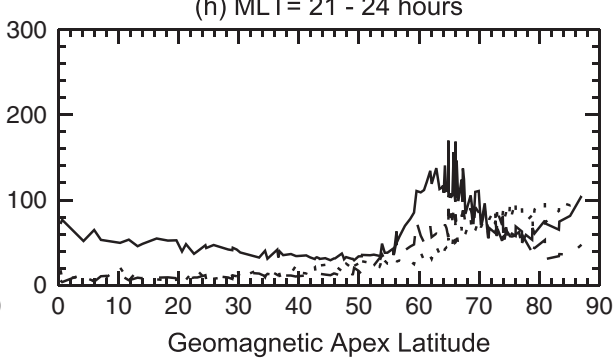

Figure 9. Mean absolute value of measured magnetic perturbations as a function of corrected geomagnetic apex latitude. The magnitude of the IMF was in the range of 12 to $20 \mathrm{nT}$, with no sorting by IMF clock angle. The entire 8 year database was used. $(\mathrm{a}-\mathrm{h})$ Results for sorting by MLT, with each bin covering $3 \mathrm{~h}$ of MLT. The three lines show the results for $\Delta B_{\text {North }}$ (solid), $\Delta B_{\text {East }}$ (dotted), and $\Delta B_{\text {Vertical }}$ (dashes). Each data point in a line corresponds to one site.

tend to be superposed over the more slowly varying, background levels that are better predicted.

[40] It is most likely that these higher frequencies are due to rapid motions of the aurora that result from chaotic, turbulent processes in the magnetotail. As the aurora moves overhead, there are momentary intensifications of the conductivities and electrojet currents. The effects are expected to be random and have magnitudes and timings that are not exactly predictable. Pulkkinen et al. [2006] had investigated the ground geomagnetic field variations and found that "the spatiotemporal behavior of the time derivative of the magnetic field above temporal scales of $100 \mathrm{~s}$ resembles that of uncorrelated white noise; this may set constraints on the achievable forecasting accuracy of the time derivative of the magnetic field." However, the empirical model results may indicate when and where the more rapid variations are likely to occur.

[41] Comparisons of model calculations with magnetometer measurements in other cases have produced similar results. In the test results presented by Pulkkinen et al. [2011], an earlier version of this model did very well in a direct comparison with other prediction methods, such as MHD simulations, with predicting the overall level of the geomagnetic perturbations. On the other hand, since the empirical model does not reproduce the rapid and chaotic temporal variations on the order 


\section{WEIMER: A MODEL OF GEOMAGNETIC PERTURBATIONS}

(a) $\mathrm{MLT}=0-3$ hours

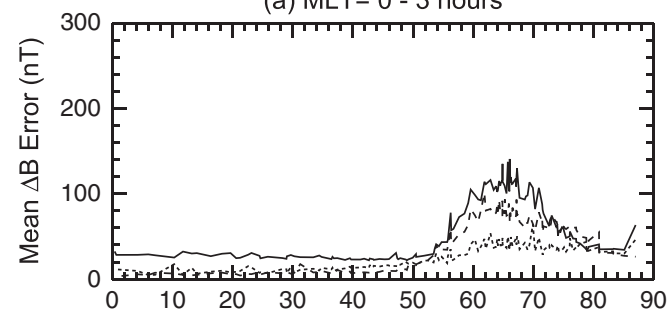

(c) $\mathrm{MLT}=6-9$ hours

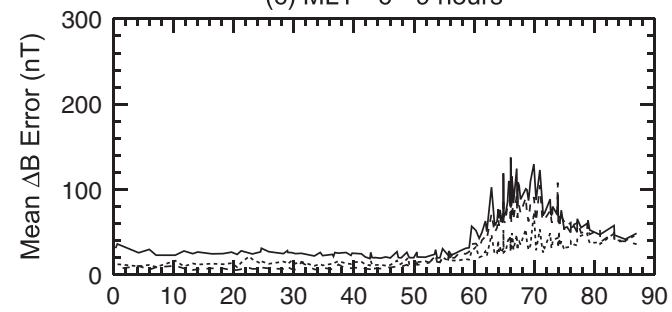

(e) $\mathrm{MLT}=12-15$ hours

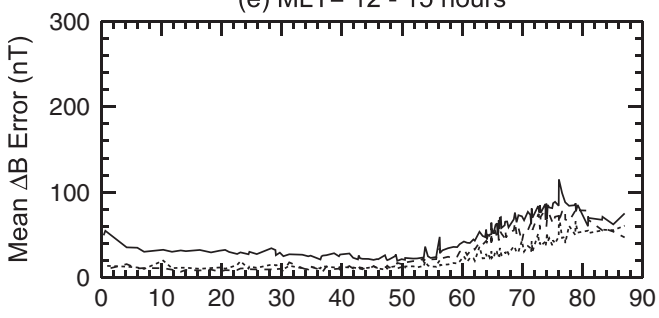

(g) $M L T=18-21$ hours

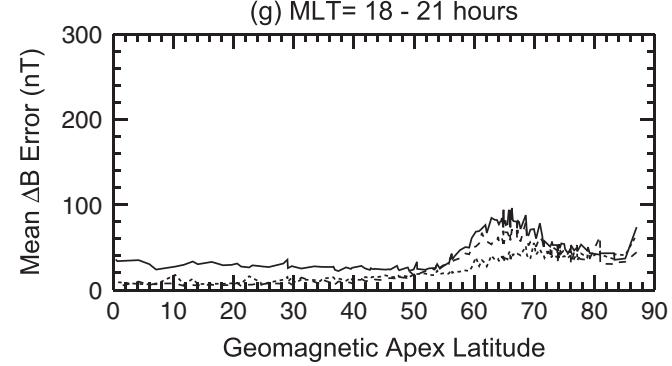

(b) $\mathrm{MLT}=3-6$ hours

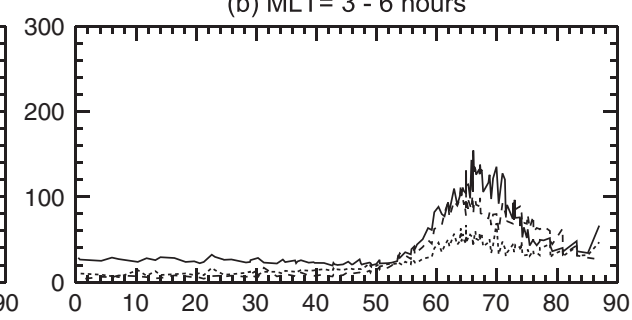

(d) $\mathrm{MLT}=9-12$ hours

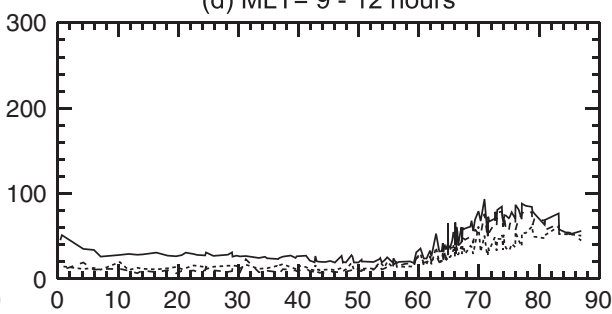

(f) $M L T=15-18$ hours

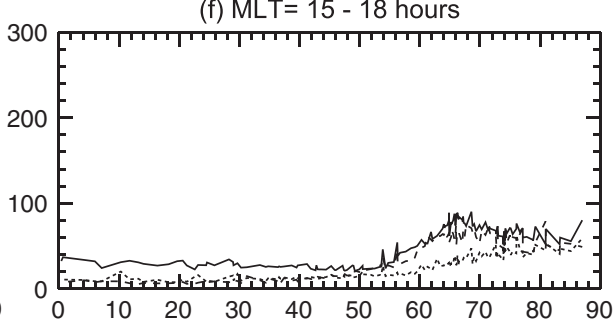

(h) $\mathrm{MLT}=21-24$ hours

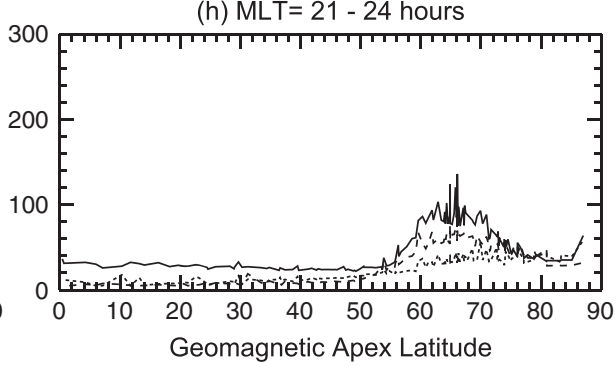

Figure 10. Mean absolute value of model errors as a function of corrected geomagnetic apex latitude. The same data and format are used as shown in Figure 9. At every 5 min time step in the 8 year period, the actual IMF, solar wind velocity, $\mathrm{F}_{10.7}$ index, and dipole tilt angle were input to the model. The differences between the model and measured magnetic perturbations were calculated at each site to obtain these means, grouped according to the stations' MLT at each time step. As in Figure 9, the three lines show the results for $\Delta B_{\text {North }}$ (solid), $\Delta B_{\text {East }}$ (dotted), and $\Delta B_{\text {Vertical }}$ (dashes).

of $1 \mathrm{~min}$, it does not do as well on tests of frequency spectra and the peak rate of change $(\mathrm{d} B / \mathrm{d} t)$ in the magnetic field. A. Pulkkinen et al. (submitted manuscript, 2012) present the results of further testing by the NASA CCMC that includes a more recent, but not final, version of this model. These tests investigated the "Probability of Detection," "Probability of False Detection," and "Heidke Skill Score" for $\mathrm{dB} / \mathrm{d} t$ in the range of 0.3 to $1.5 \mathrm{nT} / \mathrm{s}$. The scores for this empirical model were generally midrange in comparison to the other prediction models.

[42] One of the reasons for expanding the data coverage to 8 years was to obtain samples over a wide range of solar $F_{10.7}$ indices. In the results, the low-latitude, $S_{q}$ field illustrated in Figure 5 increases if the $F_{10.7}$ is made larger, as expected. As mentioned earlier, the square root of $\mathrm{F}_{10.7}$ is used in the model. Model fits that used the $\mathrm{F}_{10.7}$ index with exponents other than $1 / 2$ were tested, but use of the $1 / 2$ power produced the lowest total error. It was a very weak effect, since other variables are more dominating.

[43] At high latitudes, the effect of varying the season (dipole tilt) as well as the $\mathrm{F}_{10.7}$ index sometimes produces the expected results, where peak values are larger in magnitude in the cases with the larger solar $F_{10.7}$ index, particularly if the minimum/maximum is located on the 
day side. The greater solar ultraviolet radiation should correspond to higher ionospheric conductivities, larger currents, and greater magnetic effects seen at the ground. But it is not always the case that the magnetic perturbations are greater with the higher $\mathrm{F}_{10.7}$ index and/or positive tilt angles (summer). When comparing different cases side by side, the larger magnetic perturbations sometimes occur in the winter or with the lower $F_{10.7}$ index. The apparent contradiction may be due to inter-hemispheric effects, precipitation-enhanced conductivity, or changes in the altitude of the peak conductivity layer.

[44] The maps of the vertical component of the perturbations, shown in the right column of Figure 4, have a strong resemblance to contour maps of the corresponding fieldaligned current (FAC) density that are obtained from satellite magnetometer measurements. This feature was mentioned previously by Weimer et al. [2010]. The resemblance is seen for all IMF orientations and suggests that the vertical perturbations can be used as a "proxy" indicator of the overhead FAC.

[45] As illustrated in Figure 3, the geomagnetic perturbations on the ground do exhibit the saturation effect as the magnitude of the IMF is increased. The curve does not exactly follow the saturation that is observed in the polar electric potentials. This may be due to variations in ionospheric conductivity, including distribution with altitude. Interestingly, the positive, northward perturbations at post-noon do reach the saturation level at approximately the same point as the electric potentials, while the negative perturbations at early dawn MLT continue to rise. The reason for this disparity is not known, but the most likely possibility is that the Hall conductivity increases more in the electrojets at dawn due to particle precipitation.

[46] The geographical spacing of magnetic observatories below the equator is presently too sparse for extension of this model to the Southern Hemisphere. However, the model can still be used in that hemisphere, by reversing the input parameters for the dipole tilt angle and GSM $Y$ component of the IMF as well as reversing the sign of the east and vertical components on the output.

[47] At the present this model does not handle spatialtemporal variations that are produced by sudden pressure impulses in the solar wind nor geomagnetic substorms. Some of the more poorly matched variations seen in Figure 6 are likely due to substorm excursions, which are also a source of the higher errors seen near midnight in Figure 10. The capability to handle these temporal changes would be a useful addition in the future. There already has been some progress on substorms, as reported by Pothier et al. [2011, also submitted manuscript, Mapping groundlevel magnetic perturbations during substorms, submitted to Journal of Geophysical Research, 2012]. Using the same analysis techniques described in W10, it was found that substorms have reproducible patterns through the expansion and recovery phases, when sorted by peak substorm magnitudes, season, and the IMF orientation. What is currently lacking is a reliable way to forecast the onset times and magnitudes of substorms. If substorm expansion times can be predicted, in the future it would be possible to superimpose the mappings found by N. M. Pothier et al. (submitted manuscript, 2012) in order to improve model accuracy at the most critical times.

\section{Conclusions}

[48] A new empirical model that has the capability to predict geomagnetic perturbations at the surface of earth is described here. This program is constructed from a large database of measurements from magnetic observatories over an 8 year period, along with the simultaneous measurements in the solar wind by the ACE satellite. Although the final least-error fit calculations required several days to ingest the data, maps of the perturbations for the entire Northern Hemisphere, for all three vector components, can be computed from this model in under a second. For example, Figure 4 was drawn in $3 \mathrm{~s}$. This speed provides a considerable advantage over the numerical MHD models.

[49] Actual predictions of the geomagnetic perturbations can be obtained with an approximately $1 \mathrm{~h}$ lead time through the use of real-time measurements in the solar wind from the ACE satellite or its future replacement. A demonstration of this capability is presently shown at http://mist.nianet.org/weimerGeomag.html and has been running continuously for over a year.

[50] Using a comparison with magnetometer measurements, it is shown that the large-scale low-frequency magnetic variations are predicted quite well by the model. In addition to the fast computational times, the main advantage that this model has is that it implicitly incorporates the effects of variable ionospheric conductivity and underground image currents without the need for other models or assumptions.

[51] For the problems caused by GIC in man-made structures, the most important factors are the rate of change in the horizontal magnetic field, $\mathrm{d} B / \mathrm{d} t$, as well as the local ground conductivity near the surface. As shown by Gannon et al. [2012], some geographic regions are more susceptible to induced electric fields than others. "Hazard assessment maps" for "specific physiographic regions" are presently under development [Gannon et al., 2012].

[52] The primary weakness of this model is that it does not do as well as others on tests of $\mathrm{d} B / \mathrm{d} t$, as it does not handle the higher-frequency variations that occur over short distances. It may be that these more rapid fluctuations may be beyond the ability of any model to predict with precision, due to their random and chaotic nature. The largest variations tend to occur when and where the background level is high, so even if exact predictions cannot be made, the results from this model can indicate when and where large $\mathrm{d} B / \mathrm{d} t$ could be expected to occur, as they appear to scale with the level of the slower variations.

[53] Currently, the NOAA Space Weather Prediction Center provides forecasts of global geomagnetic activity indices, such as a $3 \mathrm{~h} \mathrm{Kp}$. Through use of an empirical model such as the one described here, it would be possible to provide more local forecasts, such as "regional K indices" at shorter time scales. 
[54] Acknowledgments. This work is funded by the National Space Weather Program through NSF grant ATM-0817751 to Virginia Tech. The author thanks the many personnel and institutions that maintain the magnetometer arrays: CARISMA, University of Alberta, and the Canadian Space Agency; the member institutes of INTERMAGNET; the Geophysical Institute of the University of Alaska; Augsburg College MACCS Project; The Danish Meteorological Institute; the Scandinavian IMAGE Array; and the University of Iceland.

\section{References}

Baker, K. B., and S. Wing (1989), A new magnetic coordinate system for conjugate studies at high latitudes, J. Geophys. Res., 94, 9139.

Boteler, D. H. (2001), Space weather effects on power systems, in Space Weather, Geophys. Monogr. Ser, vol. 125, pp. 347-352, AGU, Washington, D. C.

De Zeeuw, D. L., S. Sazykin, R. A. Wolf, T. I. Gombosi, A. J. Ridley, and G. Toth (2004), Coupling of a global MHD code and an inner magnetospheric model: Initial results, J. Geophys. Res., 109, A12219, doi:10.1029/2003JA010366.

Emmert, J. T., A. D. Richmond, and D. P. Drob (2010), A computationally compact representation of MagneticApex and QuasiDipole coordinates with smooth base vectors, J. Geophys. Res., 115, A08322, doi:10.1029/2010JA015326.

Engebretson, M. J., W. J. Hughes, J. L. Alford, E. Zesta, J. L. J. Cahill, R. L. Arnoldy, and G. D. Reeves (1995), Magnetometer array for cusp and cleft studies observations of the spatial extent of broadband ULF magnetic pulsations at cusp/cleft latitudes, J. Geophys. Res., 100, 19,371.

Fairfield, D. H. (1971), Average and unusual locations of the Earth's magnetopause and bow shock, J. Geophys. Res., 76(28), 6700.

Gannon, J. L., L. Tritchentko, and P. Fernberg (2012), United States regional GIC hazard assessment, Abstract SM21D-08 presented at 2012 Fall Meeting, AGU, San Francisco, CA, 3-7 Dec.

Gleisner, H., and H. Lundstedt (2001), A neural network-based local model for prediction of geomagnetic disturbances, J. Geophys. Res., 106(A5), 8425.

Gummow, R. A. (2002), GIC effects on pipeline corrosion control systems, J. Atmos. Sol.-Terr. Phys., 64(16), 1755, doi:10.1016/S13646826(02)00125-6.

Gustafsson, G., N. E. Papitashvili, and V. O. Papitashvili (1992), A revised corrected geomagnetic coordinate system for Epochs 1985 and 1990, J. Atmos. Sol.-Terr. Phys., 54, 1609, doi:10.1016/0021-9169(92)90167-J.

Haines, G. V. (1985), Spherical cap harmonic analysis, J. Geophys. Res., 90(B3), 2583.

Hapgood, M. A. (1992), Space physics coordinate transformations: A user guide, Planet. Space Sci., 40(5), 711-717.

Hapgood, M. A. (1997), Corrigendum, Planet. Space Sci., 45(8), 1047.

Hughes, W. J., M. J. Engebretson, and E. Zesta (1995), Ground observations of transient cusp phenomena: Initial results from MACCS, in The Physics of the Magnetopause, Geophys. Monogr. Ser, vol. 90, edited by P. Song, B. U. O. Sonnerup, and M. F. Thomsen, p. 427, AGU, Washington, D. C.

Kivelson, M. G., and A. Ridley (2008), Saturation of the polar cap potential: Inference from Alfven wing arguments, J. Geophys. Res., 113, doi:10.1029/2007JA012302.

Knecht, D. J., and B. M. Shuman (1985), The geomagnetic field, in Handbook of geophysics and the space environment, edited by A. S. Jursa, fourth ed., chap. 4, pp. 1-37, Air Force Geophysics Lab., Hanscom AFB, MA.

Langhoff, S. R., and T. Straume (2012), Highlights of the "space weather risks and society workshop", Space Weather, 10, S06004, doi:10.1029/2012SW000792.

Lanzerotti, L. J. (2001), Space weather effects on technologies, in Space Weather, Geophys. Monogr. Ser, vol. 125, edited by P. Song, H. J. Singer, and G. L. Siscoe, pp. 11-22, AGU, Washington, D.C.

Lanzerotti, L. J. (2012a), Space weather and natural hazards, Space Weather, 10, S05008, doi:10.1029/2012SW000810.

Lanzerotti, L. J. (2012b), Space weather and earth electrical currents, Space Weather, 10, S05009, doi:10.1029/2012SW000814.

Matsushita, S., and H. Maeda (1965), On the geomagnetic solar quiet daily variation field during the IGY, J. Geophys. Res., 70(11), 2535, doi:10.1029/JZ070i011p02535.
McComas, D. J., S. J. Bame, P. Barber, W. C. Feldman, J. L. Phillips, and P. Riley (1998), Solar wind electron, proton, and alpha monitor (SWEPAM) on the Advanced Composition Explorer, Space Sci. Rev., 86, 563.

Ober, D. M., G. R. Wilson, W. J. Burke, N. C. Maynard, and K. D. Siebert (2007), Magnetohydrodynamic simulations of transient transpolar potential responses to solar wind density changes, J. Geophy. Res., 112, A10212, doi:10.1029/2006JA012169.

Pirjola, R., and A. Viljanen (1998), Complex image method for calculating electric and magnetic fields produced by an auroral electrojet of finite length, Ann Geophys., 16, 1434.

Pothier, N. M., D. R. Weimer, and W. Moore (2011), Earth's ground-based magnetic perturbations in response to substorms, Abstract SA51D-1983 presented at 2011 Fall Meeting, AGU, San Francisco, CA, 5-9 Dec.

Press, W. H., S. A. Teukolsky, W. T. Vetterling, and B. P. Flannery (1992), Numerical Recipes in C: The Art of Scientific Computing, 2nd ed., pp. 43-50, Cambridge Univ. Press, New York.

Pulkkinen, A., A. Klimas, D. Vassiliadis, V. Uritsky, and E. Tanskanen (2006), Spatiotemporal scaling properties of the ground geomagnetic field variations, J. Geophys. Res., 111, A03305, doi:10.1029/ 2005JA011294.

Pulkkinen, A., M. Hesse, M. Kuznetsova, and L. Rastätter (2007), Firstprinciples modeling of geomagnetically induced electromagnetic fields and currents from upstream solar wind to the surface of the earth, Ann. Geophys., 25, 881, doi:10.5194/angeo-25-881-2007.

Pulkkinen, A., et al. (2011), Geospace Environment Modeling 2008-2009 Challenge: Ground magnetic field perturbations, Space Weather, 9, S02004, doi:10.1029/2010SW000600.

Raeder, J., et al. (2001), Global simulation of the Geospace Environment Modeling substorm challenge event, J. Geophys. Res., 106(A1), 381, doi:10.1029/2000JA000605.

Rasson, J. L. (2007), INTERMAGNET, in Encyclopedia of geomagnetism and paleomagnetism, edited by D. Gubbins, and E. Herrero-Bervera, pp. 715-717, Springer-Verlag, New York.

Richmond, A. D. (1995), Ionospheric electrodynamics using magnetic apex coordinates, J. Geomag. Geoelectr., 47, 191.

Robinson, R. M., and R. R. Vondrak (1984), Measurements of E region ionization and conductivity produced by solar illumination at high latitudes, J. Geophys. Res., 89(A6), 3951.

Siscoe, G., J. Raeder, and A. J. Ridley (2004), Transpolar potential saturation models compared, J. Geophys. Res., 109, A09,203, doi:10.1029/2003JA010318.

Siscoe, G. L., G. M. Erickson, B. U. O. Sonnerup, N. C. Maynard, J. A. Schoendorf, K. D. Siebert, D. R. Weimer, W. W. White, and G. R. Wilson (2002), Hill model of transpolar potential saturation: Comparisons with MHD simulations, J. Geophys. Res., 107(A6), 1075, doi:10.1029/2001JA000109.

Smith, C. W., M. H. Acuna, L. F. Burlaga, J. L. N. F. Ness, and J. Scheifele (1998), The ACE Magnetic Field Experiment, Space Sci. Rev., 86, 613.

Sonnerup, B. U. O. (1974), Magnetopause reconnection rate, J. Geophys. Res., 79, 1546.

St-Louis, B. (2008), INTERMAGNET technical reference manual, 4.4th ed, INTERMAGNET, Edinburgh, UK.

Stauning, P., and O. A. Troshichev (2008), Polar cap convection and PC index during sudden changes in solar wind dynamic pressure, J. Geophys. Res., 113, A08227, doi:10.1029/2007JA012783.

Tanskanen, E. I., A. Viljanen, T. I. Pulkkinen, R. Pirjola, L. Häkkinen, A. Pulkkinen, and O. Amm (2001), At substorm onset, 40\% of AL comes from underground, J. Geophys. Res., 106(A7), 13119, doi:10.1029/2000JA900135.

Tobiska, W. K., S. D. Bouwer, and B. R. Bowman (2008), The development of new solar indices for use in thermospheric density modeling, J. Atmos. Sol. Terr. Phys., 70, 803.

Turner, R. (2012), National response to a severe space weather event, Space Weather, 10, S03008, doi:10.1029/2011SW000756.

VanZandt, T. E., W. L. Clark, and J. M. Warnock (1972), Magnetic apex coordinates: A magnetic coordinate system for the ionospheric $f_{2}$ layer, J. Geophys. Res., 77, 2406.

Weigel, R. S., D. Vassiliadis, and A. J. Klimas (2002), Coupling of the solar wind to temporal fluctuations in ground magnetic fields, Geophys. Res. Lett., 29(19), 1915, doi:10.1029/2002GL014740.

Weimer, D. R. (2005), Predicting surface geomagnetic variations using ionospheric electrodynamic models, J. Geophys. Res., 110, A12307, doi:10.1029/2005JA011270. 
WEIMER: A MODEL OF GEOMAGNETIC PERTURBATIONS

Weimer, D. R., and J. H. King (2008), Improved calculations of interplanetary magnetic field phase front angles and propagation time delays, J. Geophys. Res., 113, A01105, doi:10.1029/2007JA012452. Weimer, D. R., C. R. Clauer, M. J. Engebretson, T. L. Hansen, H. Gleisner, I. Mann, and K. Yumoto (2010), Statistical maps of geomagnetic variations as a function of the interplanetary magnetic field, J. Geophys. Res., 115, A10320, doi:10.1029/2010JA015540.
Wiltberger, M., W. Wang, A. G. Burns, S. C. Solomon, J. G. Lyon, and C. C. Goodrich (2004), Initial results from the coupled magnetosphere ionosphere thermosphere model: Magnetospheric and ionospheric responses, J. Atmos. Sol. Terr. Phys., 66, 1411.

Yumoto, K., and the CPMN Group (2001), Characteristics of Pi 2 magnetic pulsations observed at the CPMN stations: A review of the STEP results, Earth Planets Space, 53, 981. 